\title{
Fuzzy-enhanced Dual-loop Control Strategy for Precise Nanopositioning
}

\author{
Mohammed Altaher \\ Centre for Applied Dynamics Research \\ University of Aberdeen \\ Aberdeen, United Kingdom \\ Email:mohammed.altaher@abdn.ac.uk
}

\author{
Sumeet S. Aphale \\ Centre for Applied Dynamics Research \\ University of Aberdeen \\ Aberdeen, United Kingdom \\ Email:s.aphale@abdn.ac.uk
}

\begin{abstract}
Vibration problems are inherent to most precision positioning systems. These systems are lightly damped and highly susceptible to mechanical resonance at any sudden change in the voltage applied to the nanopositioning platform. These systems also exhibit nonlinearity, such as hysteresis and creep. The traditional approach uses a combination of damping controllers and tracking controllers to deal with nonlinearity and resonance respectively. The classical approach is based on sole use of the integral controller (l) or proportional integral (PI) as a tracking controller to treat nonlinearity; this paper employs a hybrid feedback scheme, using the fuzzy logic controller as a correction tracking controller in in conjunction with the conventional tracking controllers. The damping controllers utilised in this work to damp the mechanical resonance of the nanopositioning platform are the Integral Resonant Controller (IRC), the Positive Velocity and Position Feedback (PVPF), and the Positive Position Feedback Controller (PPF). The proposed fuzzy logic controller delivers improved dynamic tracking performance characteristics with less vibration in comparison with the conventional tracking method because the fuzzy logic controller can handle nonlinearity and vibration via its rules and membership functions. The use of fuzzy Gaussian membership function can alleviate the appearance of mechanical resonance.
\end{abstract}

Keywords- Vibration; nonlinearity; fuzzy logic controller.

\section{INTRODUCTION}

Nanopositioning systems are lightly damped and highly susceptible to mechanical resonance when there is any sudden change in the voltage applied to the platform. The existence of nonlinearity, such as creep and hysteresis, is also a major obstacle. More robust dynamic performance can be achieved using multiple feedback loop schemes as opposed to traditional single-loop schemes. This is because singleloop feedback schemes are less sensitive to nonlinearity such as hysteresis; this can be related to the fact that the singleloop feedback makes small corrections in order to track perfectly [1].

Improving the dynamic characteristics of the piezoelectric platform is of significant importance in achieving accurate tracking performance in nanopositioning applications. One of the most important goals of the fuzzy logic controller is improving the dynamic characteristics of the system as compared with conventional controllers [2]. A comparative analysis of the fuzzy logic controller and its counterpart, the conventional controller, has proven that the fuzzy logic controller shows better dynamics behaviour [3]. Further, the fuzzy logic controller can be used in conjunction with conventional control techniques to augment them and simplify their design [4]. In the case of nonlinear dynamics or uncertainty, the fuzzy logic controller is preferred over classical control techniques. The design of the fuzzy logic controller is uncomplicated and faster than conventional controllers; it is in fact easy to implement [5].

The traditional approach uses the proportional integral (PI) or integral (I) as a tracking controller to treat nonlinearity such as creep and hysteresis, and damping controllers to treat the mechanical resonance of the platform [6]. This paper employs the fuzzy logic controller as a tracking correction controller in a multi-loop feedback scheme in conjunction with three types of damping controller. The proposed damping controllers are the Integral Resonant Controller (IRC) [7], the Polynomial Based Controller (PVPF) [8] and the Positive Position Feedback (PPF) [9].

The paper is organised as follows: Section II describes the background theory and the hysteresis nonlinearity. In Section III, tracking controllers, including the conventional and fuzzy logic controller, are explained. Fuzzy correction is introduced as a tracking controller in section IV to prove the effectiveness of the proposed hybrid scheme and the damping controllers are presented in Section V. Section VI provides results to validate the enhanced performance of the scheme and section VII concludes the paper.

\section{System Modelling}

The dynamics of the nano-axis has linear and nonlinear components; thus, the axis is modelled as a linear secondorder transfer function and a Bouc Wen model for hysteresis.

\section{A. Linear Dynamics Model}

The mechanical system of the nanopositioning platform is shown in figure 1(a) [10], which can be characterised and simplified by a spring-damper system, as shown in figure 1(b) [11]. The axis of the nanopositioning platform is equipped with a capacitive sensor for position measurement. The equation of motion for this system is given by:

$$
M_{p} \ddot{d}+c_{f} \dot{d}+\left(K_{a}+k_{f}\right) d=F_{a}
$$


The system dynamics is regulated by the piezoelectric actuator force that moves the nanopositioning stage. The movement of the piezo actuators is manifested by expansion and contraction in response to an input voltage stimulus. Thus, $\left(F_{s}\right)$ is the measured force acting between the actuator and the mass of the platform $\left(M_{p}\right)$ in the vertical direction. The stiffness of the actuator is denoted by $\left(K_{a}\right)$ and the force by $\left(F_{a}\right)$. A force sensor is collocated with the actuator and measures the load force $\left(F_{s}\right)$. Equation 1 can now be rewritten as follows:

$$
M_{p} \ddot{d}+c_{f} \dot{d}+k_{d}=F_{a}
$$

The relationship between the applied force $\left(F_{a}\right)$ and the

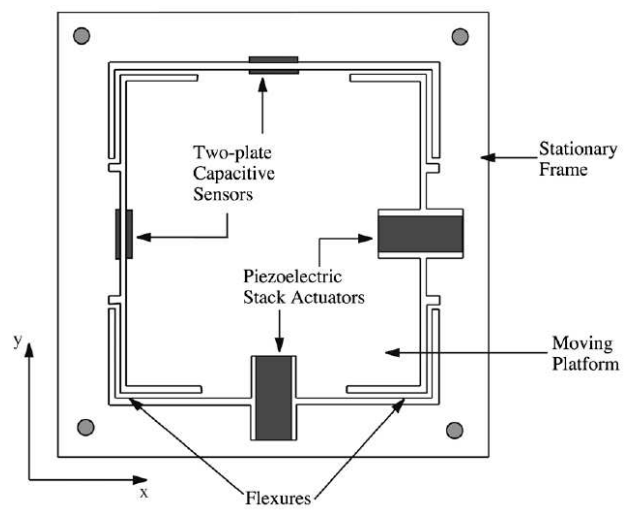

(a)

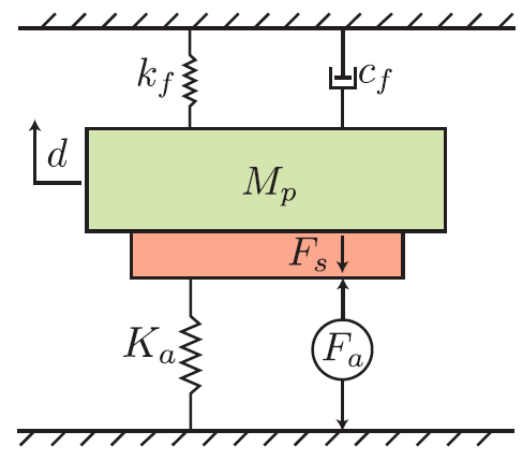

(b)

Figure 1. a- A simple schematic of a piezo-stack actuated two-axis nanopositioner; $b$ - The equivalent mass-spring-damper model for one axis of the nanopositioner

displacement $(d)$ is described as in the following transfer function:

$$
G_{d F_{a}}(s)=\frac{d}{F_{a}}=\frac{1}{M_{p} s^{2}+c_{f} s+k}
$$

In a nanopositioning system, the transfer function of the plant is identified using the frequency response analysis in analogy with (3). The transfer function is represented by a second order model, as in the equation below:

$$
G(s)=\frac{\sigma^{2}}{s^{2}+2 \zeta \omega_{n} s+\omega_{n}^{2}},
$$

where $\zeta$ is the damping ratio, $\omega_{n}$ is the natural frequency, and $\sigma^{2}$ is selected to manipulate the DC gain of the platform. This representation is valid only if the first dominant mode is considered. In the event that more than one mode is considered, the overall transfer function for the piezoelectric platform can be represented by the summation of several second order systems for each mode. For reasons of practicality, other modes can be truncated.

According to previous research, the transfer function of the platform has been identified solely based on the first dominant mode [12] and given by:

$$
\frac{2.025 * 10^{7}}{s^{2}+48.63 s+1.042 * 10^{7}}
$$

\section{B. Hysteresis Model}

Nonlinear effects are usually unmodelled and tracking is enforced in order to minimise the effect of nonlinearities on the actual trace. Hysteresis is a dynamic characteristic present in many physical systems, such as piezo actuators. Hysteresis in piezo actuators can lead to problems such as an increase in undesirable inaccuracy or oscillation and instability [13]. Therefore, any control strategy must be designed to accommodate uncertain time-varying nonlinear systems. Since the piezoelectric platform (nanopositioning system) is a nonlinear system, the hysteresis has been modelled using the Bouc Wen [14]. The nanopositioning platform can be represented as a mass-spring damper and the relationship between the applied voltage and the displacement is nonlinear. The equation of motion for the piezoelectric platform can be described using the Bouc Wen through nonlinear differential equations, as in (6) [15]:

$$
\left\{\begin{array}{l}
m \ddot{x}+b \dot{x}+k x=k(d u-h) \\
\dot{h}=\alpha d \dot{u}-\beta|\dot{u}| h-\gamma \dot{u}|h|
\end{array}\right\}
$$

where $\mathrm{h}$ represents the nonlinear relation between the lag force and the displacement. The parameters $\alpha, \beta$ and $\gamma$ have been identified in order to represent the hysteresis loop's magnitude and shape, as in (7):

$$
\left\{\begin{array}{c}
\alpha=0.26 \\
\beta=0.005 \\
\gamma=0.00068 \\
\mathrm{~d}=2 \mu \text { m per volt }
\end{array}\right\}
$$

The applied voltage can be denoted as $\mathrm{u}$, and $\mathrm{x}$ as the displacement of the piezoelectric actuator; $m, b, k$ and $d$ represent the effective mass, damping coefficient, mechanical stiffness and effective piezoelectric coefficients respectively. The selection of the Bouc Wen model, in preference to other models was due to its simplicity and ability to capture the 
major hysteresis cycles. The hysteresis has been realised in MATLAB using a nonlinear differential equation representation of the Bouc Wen simulated in Simulink to describe the hysteresis.

The open-loop investigation of the modelling of hysteresis is presented in figure 2(a) and (b), which is associated with a single axis of the nanopositioning platform. The deviation from linearity in tracking the triangle wave is clearly depicted in figure 2(a) in the open-loop. The proposed hysteresis model is investigated by applying a 50 volt peak amplitude sinusoidal signal of $10 \mathrm{~Hz}$ to the platform and the hysteresis cycle is thereby generated as is clear in figure 2 (b). A nonlinear relationship was found to exist between the control voltages applied on the piezoelectric and its displacements. The generated displacements were found to be different for the forward and backward paths under the same voltage and the induced nonlinearity hysteresis noted displayed $10 \mu \mathrm{m}$ of lag in the displacement, as shown in figure 2(b).

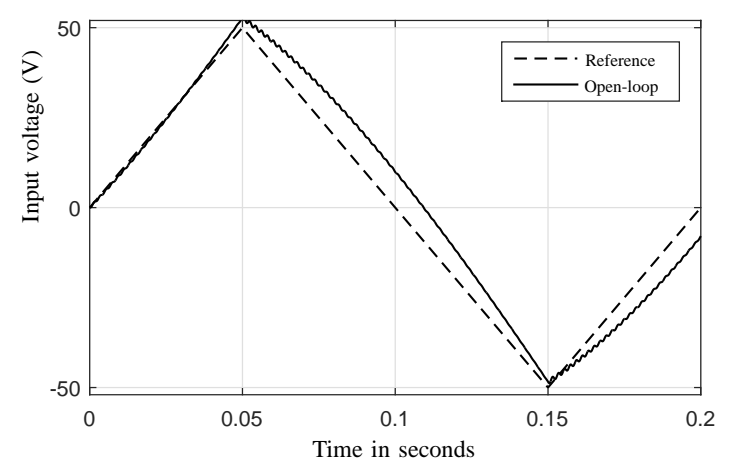

(a)

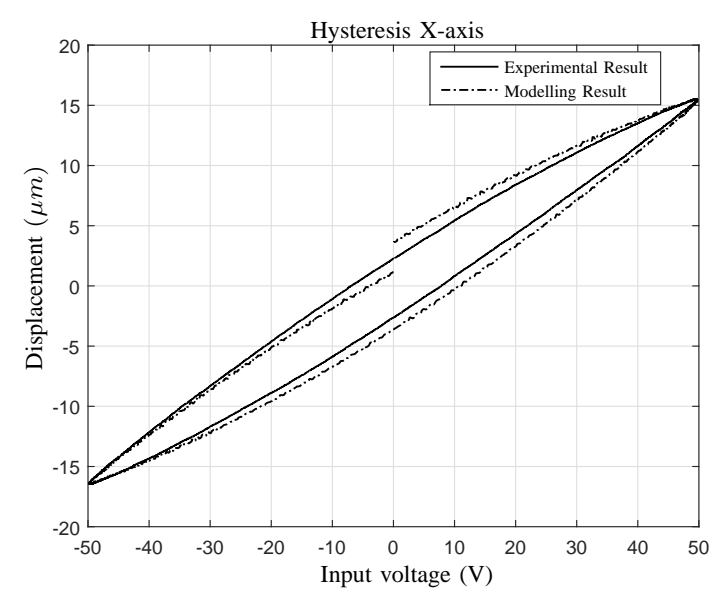

(b)

Figure 2. a-Illustrates the deviation from linearity in the open-loop in tracking triangle wave of $5 \mathrm{~Hz}$; b- Measured and modelled hysteresis loops show that the hysteresis model accurately captures the hysteresis of the piezo actuator

In figure 2(b), a comparison can be seen between the experimental and the modelling result, where the $\mathrm{x}$-axis represents the input voltage and the y-axis is the generated displacement. It can be seen from figure 2(b) that the openloop exhibits strong nonlinearity. A system exhibiting such hysteresis is severely limited in its performance. The hysteresis loop provides a rate-independent relationship between the applied voltage and the generated displacement.

The following section aims to briefly explain the principle operation of tracking controllers and damping.

\section{TRACKING CONTROLLER}

\section{A. Conventional Tracking Controllers}

Controllability of the nanopositioning platform is a challenging and difficult matter due to various control problems associated with the platform; hence a combination of different controllers is required. The initial approach in controlling the platform is to use a suitable damping controller for resonance and a well-designed tracking controller. There are different types of damping and tracking controllers, as will be explained later in this paper. Figure 3 illustrates the method by which the tracking and damping controllers are combined and used together.

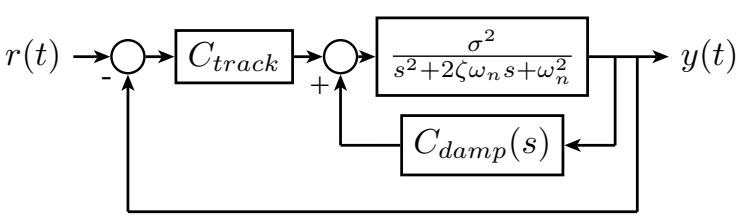

Figure 3. Schematic diagram illustrating the traditional distribution of damping-tracking controllers

In nanopositioning, the classical tracking controllers are generally either proportional-integral (PI) or integral (I). The conventional tracking controller that has been used in conjunction with all the damping controllers is a simple integrator with a transfer function given by:

$$
C_{\text {track }}(s)=\frac{k_{t}}{s}
$$

This work has thus far focused on linear controllers; however, nonlinear controllers have begun to be implemented in many studies. This research will investigate a nonlinear control strategy for better performance. The fuzzy logic controller will be used as a tracking controller to mimic the integral action, as will be explained in the following section. This paper proposes the fuzzy like PI, as the nonlinear tracking controller in conjunction with damping controllers instead of a conventional (PI) or (I) tracking controller.

\section{B. Fuzzy Logic Tracking Controller}

The fuzzy system is knowledge-based and human experience can be incorporated into its design by mimicking expert knowledge via the fuzzy rules [16]. 
In this work, the rules have been designed based on an analogy of the classical control system modelling. The dynamic characteristics of the proposed control system have been considered as the fuzzy model and the linguistic description of the dynamic characteristics can be used to obtain a set of fuzzy rules. The conventional PI control law is given by:

$$
U(t)=K_{p} e(t)+K_{i} \int e(t) d t
$$

where $e(t)$ is the error as function of time, $K_{i}$ is the integral gain, $U(t)$ is the control output as function of time, $K_{p}$ is proportional gain and $T_{i}$ is an integral constant. The fuzzy logic incremental controller can be written as:

$$
\nabla U(k)=K_{p} \nabla e(k)+K_{i} e(k)
$$

The incremental fuzzy controller is a type of fuzzy logic control system whereby the controller output is not the control action $U$, but instead the change of the control action $\nabla U$. An incremental controller adds a change in the control signal $\nabla U$ to the current control signal, as in (11):

$$
U(K)=U(k-1)+\nabla U .
$$

In figure 4 a clear illustration of the fuzzy logic controller gains' distribution is presented. From the figure, it can be seen that the fuzzy logic controller will generate $\nabla U$ as the nonlinear function of normalised error and normalised change of error.

$$
\nabla U_{n}(k)=f\left(e_{N}, \nabla e_{N}\right)
$$

The actual output of the fuzzy logic controller is a nonlinear function of error and change of error. The function $f$ is the input-output map of the fuzzy logic controller, as is clear from (13):

$$
\nabla U(k)=f(G E * e, G C E * \nabla e)
$$

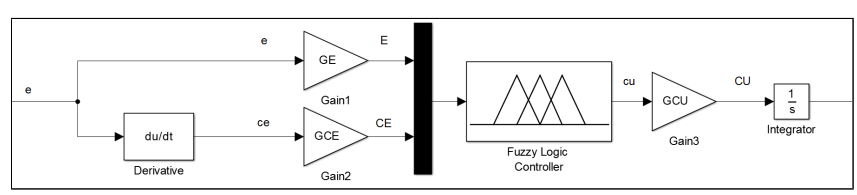

Figure 4. Fuzzy incremental controller

The relationship between the scaling factors for the fuzzy logic controller and the conventional controller has been derived based on an analogy of the conventional controller. It has been found that $G C E$ multiplied by $G C U$ is equivalent to $K_{p}$ and $\frac{G E}{G C E}$ is equivalent to $\frac{1}{T_{i}}$. In this design the fuzzy is imitating the integral action only, hence the $G C E$ gain has been set to a very small value.

The behaviour of the fuzzy logic controller is highly restricted by its control table decisions. It can be seen from figure 5(a) that the proposed controller was formed using nine rules in order to reduce complexity. The fuzzy rules are linguistic rules characterised by and linked with conditional statements of 'IF-THEN'. These sets of linguistic conditional statements represent the control situations.

1. If (Error is N) and (change of Error is N) then (increment Control is NB) (1) 2. If (Error is N) and (change_of_Error is Z) then (increment_Control is NS) (1) 3. If (Error is Z) and (change of Error is N) then (increment_Control is NS) (1) 4. If (Error is N) and (change_of_Error is P) then (increment_Control is Z) (1) 5. If (Error is Z) and (change_of_Error is Z) then (increment_Control is Z) (1) 6. If (Error is $\mathrm{P}$ ) and (change of Error is $\mathrm{N}$ ) then (increment Control is Z) (1) 7. If (Error is Z) and (change_of_Error is P) then (increment_Control is PS) (1) 8. If (Error is P) and (change_of_Error is Z) then (increment_Control is PS) (1) 9. If (Error is P) and (change_of_Error is P) then (increment_Control is PB) (1)

(a)

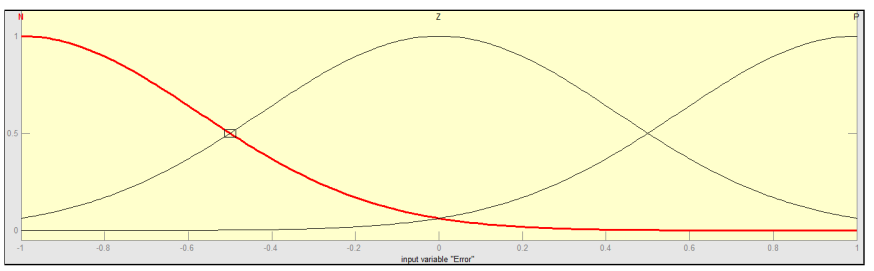

(b)

Figure 5. a-Fuzzy rules; b- Membership function of the proposed fuzzy logic controller

The control input variables (crisp inputs) must be fuzzified before applying them to the control algorithm of the fuzzy logic controller. Normally, inputs to the fuzzy logic controller (state variables) include the error and change of error. In order for the controller to continuously account for the universe of discourse, a functional definition can be used to clarify the membership function of a fuzzy set. There are several types of membership function used in this regard, such as triangular, trapezoidal and bell shaped functions. These membership functions are the most popular types in many engineering applications [17]. A Gaussian wave has been used in this design, as can be seen in figure 5(b). The proposed membership function in figure 5(b) is a plot of function $\mu$ versus $e(t)$ where the horizontal $\mathrm{x}$ axis represents the universe of discourse that covers the entire range of possible values for a chosen variable, whilst the vertical axis y represents the membership value of the fuzzy set. When implementing the membership function, the universe of discourse for the fuzzy logic controller has been normalised within $[-1,1]$ for the leftmost and rightmost respectively. Further, the membership function of the fuzzy logic controller has been designed to saturate at a peak of [+1]. Gaussian membership functions are fuzzy membership functions that represent the linguistic terms; these functions are relativity popular in the fuzzy logic literature and the output is very smooth. Hence, in this design it was decided to use the Gaussian membership function to achieve a smoother control action and avoid the abrupt change in the controller action. The reason for this is that the Gaussian membership functions are smooth and non-zero at all points. 
Using the triangle membership function would lead the fuzzy logic controller to mimic the behaviour of the conventional controller due to discontinuities in their derivatives.

In the fuzzy logic, membership is a matter of degree; it varies from 0 to 1 depending on the percentage allocated to each control situation. The vertical axis represents certainty and the horizontal axis is referred to as the universe of discourse for the input $e(t)$ since it provides the range of values of $e(t)$ that can be quantified linguistically. Normally, the crisp values are normalised between -1 and 1 for simplicity reasons. The function $\mu$ quantifies the certainty that $e(t)$ can be classified linguistically as zero. Certainty here refers to the degree of truth.

The overlap of the membership has been considered to be $25 \%$ and the membership function at the overlap is equal to 0.5; this will keep the control action smooth in the case of a sudden change. Figure 5(b) shows the implementation of the membership function for the error and change of error using three linguistic descriptions. Five linguistic descriptions have been used to quantify the control action (NB, NS, Z, PS and PB), as is clear in the Table I. It must be kept in mind that the membership functions for error, rate of error and control action have been selected to be the same (Gaussian).

Table I

THE LINGUISTICS OF THE PROPOSED FUZZY LOGIC CONTROLLER

\begin{tabular}{|c|c|c|c|c|c|}
\hline Abbreviation & NB & NS & ZE & PB & PS \\
\hline Linguistics & Negative Big & Negative Small & Zero & Positive Big & Positive Small \\
\hline
\end{tabular}

The Mamdani inference and centroid defuzzification are used in this work. Here, error (difference between set point and actual process value) and change in error (difference between current and past error) are the inputs to the fuzzy system. In the case of the fuzzy rule, the conditions can also be partially satisfied (as opposed to crisp rules); this has the desired effect of being able to interpolate between two rule conditions and achieving a smooth transition from one state to the other in the induced fuzzy control surface.

Scaling factors are important parameters to tune into the fuzzy logic controller as they can scale the universe of discourse. Changing the scaling factors will affect the meaning of the linguistics that forms the basis of the fuzzy logic controller definition, because it is redefining the horizontal axis. It can be concluded that scaling factors play a similar role to the gains of the conventional controller; they can also be a source of oscillation problems. Although selection of suitable values of scaling factors was used the trial and error method, this has been ruled out and the relationship between scaling factors and their conventional counterpart has been derived [18]. The main advantages of the fuzzy incremental control are smooth control action and removal of the steady state error. However, the disadvantage is that the controller is slow.

\section{Control Strategy}

The control object in nanopositioning applications is there to keep the tracking error to a minimum. Feedback control in applications requiring high linearity, long term position stability, repeatability and accuracy, is crucial. Traditionally, tracking, nanopositioning tracking is achieved through the use of single-loop feedback, as is clear in figure 3. Although the single-loop feedback control technique can improve the accuracy and dynamic response of the piezoelectric actuators used in nanopositioning systems, the use of feedback control law is limited in its performance [19]. In order to overcome this problem, analysis of the multi-loop feedback scheme is proposed in this paper. Hence, in this design the feedback does not flow through a single-loop. Exploration of the multi-loop closed-loop system is explained here. In order to draw a comparison with a single-loop feedback controller, the multi-loop feedback control system is described in figure 6.

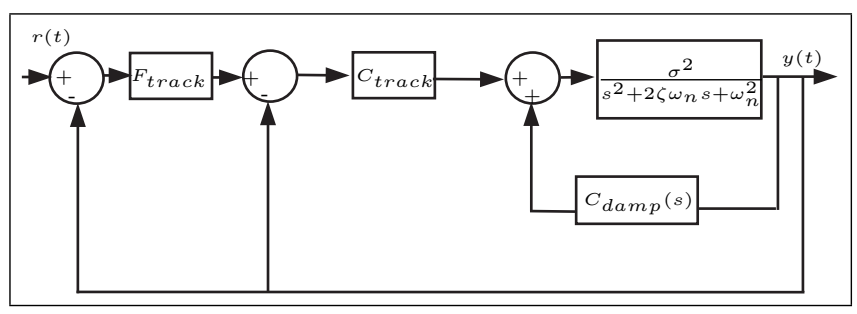

Figure 6. Proposed multi-loop fuzzy correction feedback scheme

The overall control algorithm consists of two controllers for tracking: the outer-loop uses the fuzzy logic controller $\left(F_{\text {track }}\right)$ and the inner-loop feedback use a first-order integral tracking $\left(C_{\text {track }}\right)$.

It could be argued that the fuzzy logic controller as a tracking can be used in the inner-loop feedback. Use of it in the inner-loop, however, has yet to be justified due to improvement in phase shift profile if it is used in the outerloop. The fuzzy logic controller could be applied to both loops to provide comparable results, yet to be studied. As aforementioned, the fuzzy logic controller is preferred as opposed to traditional controller in nanopositioning systems. This is due to the fact the system (piezoelectric platform) experiences the resonant mode inherently, and so the classical control will have clear limitations in damping the transient resonant mode, particularly given the fast response of the piezoelectric dynamics characteristics. The transient resonant mode will cause vibrations in terms of overshoot at any sudden change of the input signal. The occurrence of the transient resonant mode, in terms of the overshoot generated at the sudden change in the input signal, will lead to inaccuracy in the nanopositioning application. The fuzzy logic controller can improve the dynamic characteristics in terms of reducing the overshoot. 
For a typical nanopositioner, the damping ratio $(\zeta)$ is around 0.001 ; this is an extreme constraint to accurate tracking performance in nanopositioning. Therefore, the use of damping controllers is necessary. Although increasing the tracking gain to the maximum allowable value will reduce the maximum error, this reduction comes at the expense of vibration. Therefore, there is still a need for damping controllers due to the fact that the nanopositioning platforms are lightly damped. This is despite the fact that the proposed control strategy has a proven significant increase in the closed-loop bandwidth for the tracking gain.

\section{DAMPING CONTROLleRS}

\section{A. $I R C$}

The IRC controller is an effective and successful scheme for vibration damping and it can be used in a wide range of nanopositioner applications [20]. This also has the desirable property of not exciting the higher frequency dynamics [21]. The IRC can provide the appropriate damping to the system without providing tracking performance.

The IRC scheme consists of a feed-through term and integral in the feedback, as is clear in figure 7. The addition of the feed-through term provides the capacity to introduce zero that can be placed at the desired frequency. The IRC can exploit the interlacing property by introducing integral feedback, which can result in changing the phase of the system. The new phase loop lies between -90 and 90 degrees, providing the capacity for a 90 degree phase margin and infinite gain margin. According to previous research [12], the

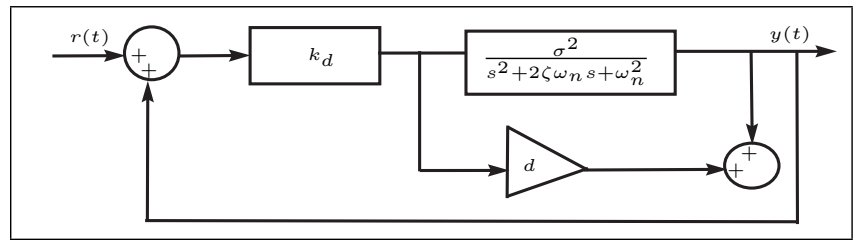

Figure 7. Schematic diagram for the IRC

value of the feed-through term (d) for the proposed system can be determined numerically. The research has also found the relationship between the tracking gain and the damping gain to rule out the trial and error method to find the optimal values for tracking and damping gains.

\section{B. PVPF Damping Control Design}

The PVPF is a control technique based on a second order transfer function in a positive feedback for active damping of the resonant mode of the system. Damping can be achieved by placing the poles of the closed-loop system at any desired location. The closed-loop poles of the lightly damped system can be shifted at the desired location to the left side of the S-plane by 1,000 units or by any arbitrary amount (this distance is used as common practice) [22]. It is important to note that the addition of the tracking controller will change the chosen locations of these poles. In fact, the real part of the open-loop poles can be sufficiently reduced to increase the damping ratio and at the same time maintain the natural frequency of the system [23]. The transfer function for the proposed controller can be written as:

$$
C_{\text {Damp-PVPF }}(s)=\frac{\Gamma_{2} s+\Gamma_{1}}{s^{2}+2 \gamma \omega_{c} s+\omega_{c}^{2}} .
$$

Figure 8 illustrates the way in which the PVPF is applied to the plant. The root locus investigation into the transfer function of the proposed system has determined the maximum allowable tracking gain in order for the system to become stable. A trial and error method has been used to examine the performance of the controller, resulting in choosing the value of the tracking gain to provide high tracking performance by increasing the linear part required to obtain accurate scanning.

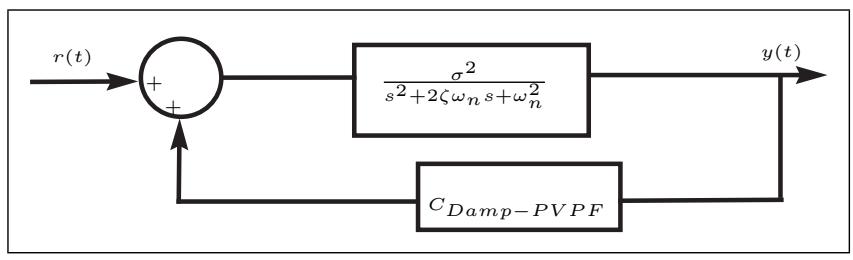

Figure 8. Schematic diagram for the PVPF

\section{PPF Design}

PPF is a second-order damping controller (filter) in a positive feedback and its transfer function has a roll off of $40 \mathrm{DB} /$ decade at higher frequencies. The simplicity of this controller and its ease of implementation are important features in making it one of the most popular control methods [24]. In the same manner as PVPF, the PPF can be designed with the transfer function given by:

$$
C_{D a m p-P P F}(s)=\frac{\Gamma_{1}}{s^{2}+2 \zeta \omega_{c} s+\omega_{c}^{2}}
$$

The fashion in which PPF is applied is clear in figure 9.

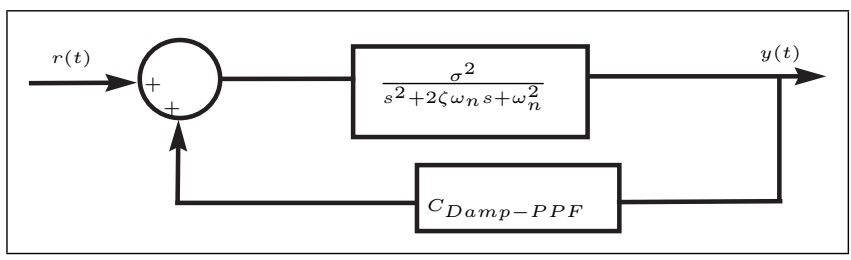

Figure 9. Schematic diagram for the PPF

Common practice in designing the PPF is based on the pole placement algorithm; the placement can be chosen arbitrarily. The controller can then be derived by equating the coefficients of the desired characteristic equation with those of the denominator of the closed-loop transfer function, and solving for the controller parameters. 


\section{RESULTS AND Discussion}

The simulation work has been undertaken using the MATLAB Simulink environment. The sampling time has been selected as $50 \mu \mathrm{s}$ microseconds due to the fast dynamic response of the piezoelectric.

There is a need to use a triangle wave as an input to the nanopositioning systems in order to obtain a raster scan. The general procedures used to achieve the raster scan involve applying triangle or square wave in the $\mathrm{x}$-axis and ramp (staircase) in the $y$ direction. In order to investigate the performance of the hybrid feedback scheme, a triangle wave has been applied to the $\mathrm{x}$-axis of the nanopositioning platform. The triangle wave has an amplitude of $100 \mu \mathrm{m}$ and a frequency of $5 \mathrm{~Hz}$. At the steady state the error has been plotted for one period for the purpose of a comparative study. The three types of damping controllers used to demonstrate the effectiveness of the hybrid feedback scheme are IRC, PVPF and PPF.

In designing the feedback schemes, it has been assumed that $1 \%$ deviation from the required linear trajectory is acceptable. Therefore, the maximum error where the tracking error is within $1 \%$ of desired trajectory is considered in the case of the hybrid feedback scheme and its counterpart error in the classical feedback scheme. This is because the area of interest for the raster scanning lies within this range. The tracking performance (time-domain and errors) for the closed-loop are plotted in figure 10(a), (b) and (c). Each figure presents a comparison of the tracking performances between the classical and fuzzy logic controller feedback schemes in conjunction with the three different types of damping controllers (IRC, PVPF and PPF).

It is important to note that increasing the tracking gain in the traditional feedback scheme will not lead to any increase in the linear region of the output trajectory. On examination of the system performance, it was calculated that the hybrid feedback scheme can increase the linearity without fear of experiencing any resonance.

It is seen that the hybrid fuzzy logic controller scheme delivers a highly linear positioning performance. As can be seen from Table II, the IRC+track delivers a $68 \%$ linear trajectory, where the fuzzy+track delivers $94 \%$. Looking at Table II, it has been observed that in the case of $5 \mathrm{~Hz}$, the feedback scheme with fuzzy correction can cope with an error of $1 \mu \mathrm{m}$ for $93 \%$ and $94 \%$ in the case of PVPF and PPF respectively, as opposed to an error of $3 \mu \mathrm{m}$. The classical feedback scheme has not shown noticeable compensation for hysteresis, whilst the hybrid scheme has remained linear even with the existence of hysteresis for a higher frequency of more than $5 \mathrm{~Hz}$.

It can be concluded that the hybrid scheme has provided approximately the same level of compensation for hysteresis using different types of damping controller. The classical feedback scheme has shown the use of PVPF and PPF better than the IRC as is evident when the error is reduced (in the case of PVPF and PPF) and the straight line in the tracking performance has occurred even for a relatively small area. It can be concluded that in the case of IRC, the classical feedback scheme cannot manage a permissible error of $1 \%$ with a straight line linearity.

Table II

TABULATES THE ENHANCED PERFORMANCE OF THE NANOPOSITIONING PLATFORM WITH HYSTERESIS

\begin{tabular}{|l|l|l|l|}
\hline \multicolumn{5}{|c|}{ Without Fuzzy Correction (IRC) Case } \\
\hline $\begin{array}{l}\text { Frequency } \\
(\mathrm{Hz})\end{array}$ & $\begin{array}{l}\text { RMS Error } \\
(\mu \mathrm{m})\end{array}$ & $\begin{array}{l}\text { Maximum Error in the Linear } \\
\text { Region }(\mu \mathrm{m})\end{array}$ & $\begin{array}{l}\text { Linearity } \\
1 \%\end{array}$ \\
\hline 5 & 1.7032 & 3.8 & $68 \%$ \\
\hline \multicolumn{5}{|c|}{ With Fuzzy Correction (IRC) Case } \\
\hline $\begin{array}{l}\text { Frequency } \\
(\mathrm{Hz})\end{array}$ & $\begin{array}{l}\text { RMS Error } \\
(\mu \mathrm{m})\end{array}$ & $\begin{array}{l}\text { Maximum Error in the Linear } \\
\text { Region }(\mu \mathrm{m})\end{array}$ & $\begin{array}{l}\text { Linearity } \\
1 \%\end{array}$ \\
\hline 5 & 0.9327 & 1 & $94 \%$ \\
\hline \multicolumn{5}{|c|}{ Without Fuzzy Correction (PVPF) Case } \\
\hline $\begin{array}{l}\text { Frequency } \\
(\mathrm{Hz})\end{array}$ & $\begin{array}{l}\text { RMS Error } \\
(\mu \mathrm{m})\end{array}$ & $\begin{array}{l}\text { Maximum Error in the Linear } \\
\text { Region }(\mu \mathrm{m})\end{array}$ & $\begin{array}{l}\text { Linearity } \\
1 \%\end{array}$ \\
\hline 5 & 0.8195 & 3 & $87 \%$ \\
\hline \multicolumn{5}{|c|}{ With Fuzzy Correction (PVPF) Case } \\
\hline $\begin{array}{l}\text { Frequency } \\
(\mathrm{Hz})\end{array}$ & $\begin{array}{l}\text { RMS Error } \\
(\mu \mathrm{m})\end{array}$ & $\begin{array}{l}\text { Maximum Error in the Linear } \\
\text { Region }(\mu \mathrm{m})\end{array}$ & $\begin{array}{l}\text { Linearity } \\
1 \%\end{array}$ \\
\hline 5 & 0.4140 & 1 & $93 \%$ \\
\hline \multicolumn{5}{|c|}{ Without Fuzzy Correction (PPF) Case } \\
\hline $\begin{array}{l}\text { Frequency } \\
(\mathrm{Hz})\end{array}$ & $\begin{array}{l}\text { RMS Error } \\
(\mu \mathrm{m})\end{array}$ & $\begin{array}{l}\text { Maximum Error in the Linear } \\
\text { Region }(\mu m)\end{array}$ & $\begin{array}{l}\text { Linearity } \\
1 \%\end{array}$ \\
\hline 5 & 0.8188 & 3 \\
\hline \multicolumn{5}{|c|}{ With Fuzzy Correction (PPF) Case } \\
\hline $\begin{array}{l}\text { Frequency } \\
(\mathrm{Hz})\end{array}$ & $\begin{array}{l}\text { RMS Error } \\
(\mu \mathrm{m})\end{array}$ & $\begin{array}{l}\text { Maximum Error in the Linear } \\
\text { Region }(\mu \mathrm{m})\end{array}$ & $\begin{array}{l}\text { Linearity } \\
1 \%\end{array}$ \\
\hline 5 & 0.3752 & 1 \\
\hline
\end{tabular}

The fuzzy logic controller has delivered improved dynamic tracking performance characteristics with less vibration in comparison with the conventional tracking method because it can cope with nonlinearity and vibration via its rules and membership functions. For example, the use of the Gaussian membership function has reduced the appearance of the mechanical resonance. Therefore, the fuzzy logic controller has been used in this design due to the capacity of its nonlinearity (fuzzy rules) to offer better dynamic characteristics.

It is noted that due to the fact that the hysteresis of the piezoelectric actuators used in nanopositioning is amplitude dependent, it exhibits highly nonlinear behaviour when a high amplitude of input voltage is applied. Since the applied voltage has a high amplitude, the classical control has clear limitations in its ability to track the reference perfectly. It could be argued that reducing the amplitude voltage can lead to decreasing the hysteresis behaviour, however, this comes at the expense of reducing the scanning area. Although flexures can be used to enlarge the movement of the piezoelectric, it is limited in its enlargement. For example the use of $1 \mu \mathrm{m}$ amplitude can be enlarged to $10 \mu \mathrm{m}$. This is resolved by using the hybrid fuzzy logic controller feedback scheme as the preferred method to overcome the amplitudehysteresis problem. 

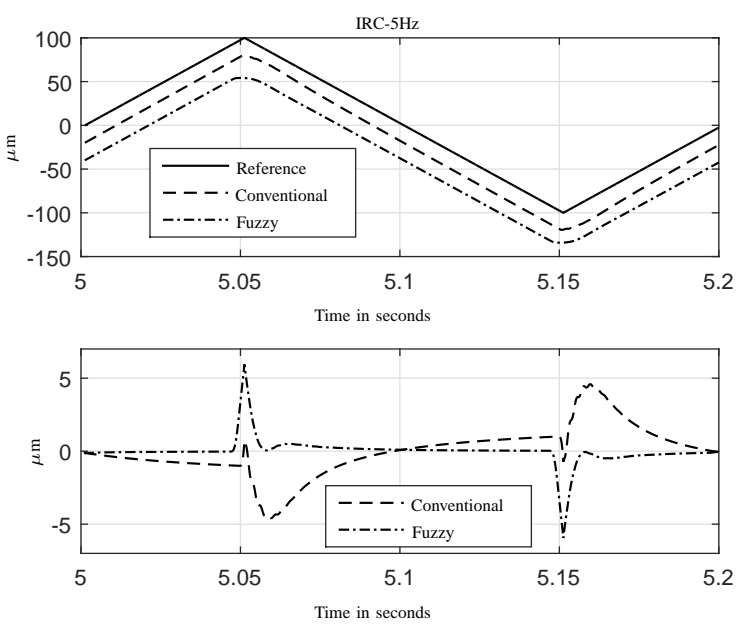

(a)
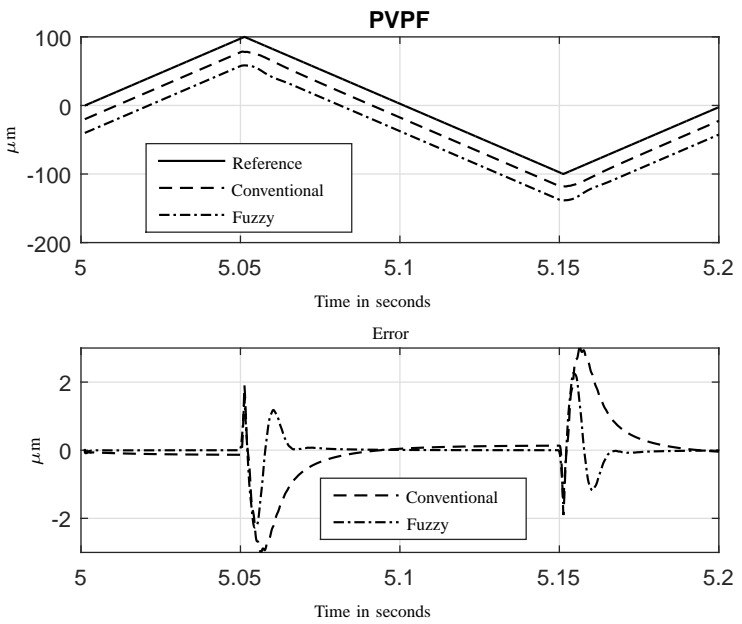

(b)
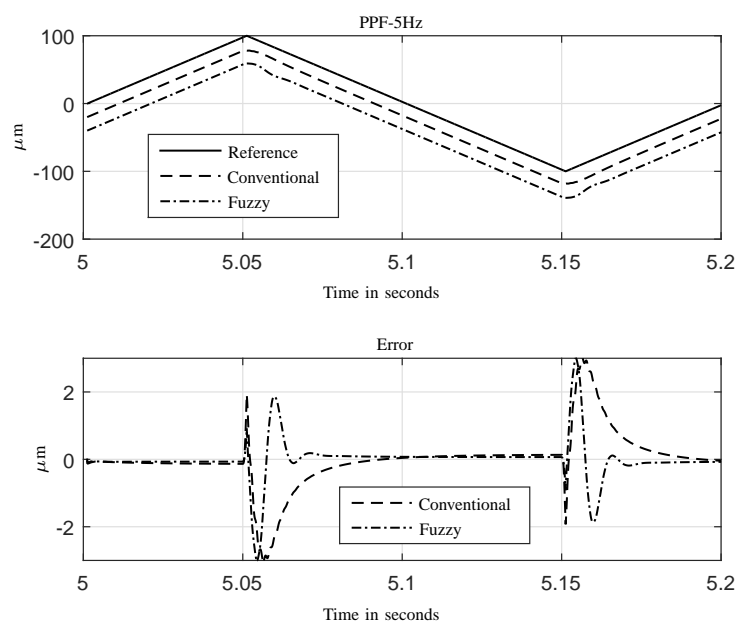

(c)

Figure 10. Comparison of the tracking performances for closed-loop between the hybrid feedback and conventional schemes; a-Time-domain tracking performance and error for one period of $5 \mathrm{~Hz}$-triangle waveform (IRC); b-Time-domain tracking performance and error for one period of $5 \mathrm{~Hz}$-triangle waveform (PVPF) c-Time-domain tracking performance and error for one period of $5 \mathrm{~Hz}$-triangle waveform (PPF) 


\section{CONCLUSION}

The main contribution of this paper is to develop a hybrid multi-loop feedback scheme with fuzzy logic controller that can compensate for nonlinear hysteresis at the precision positioning stage. The proposed hybrid multi-loop feedback scheme with fuzzy logic controller has overcome the inherent harmful nonlinearity hysteresis and enhanced the tracking performance of the nanopositioning piezoelectric actuator. The paper has introduced a new loop to be used as new input in order to introduce the output in such a way that it is closer to the desired pattern. A fuzzy-PI-controller has been developed analogously with a conventional controller and based on its rules the dynamics characteristics have been improved. According to the simulation results, the developed scheme has a verified effective solution to better compensation for hysteresis and the results have provided a satisfactory result in terms of the induced error generated.

\section{REFERENCES}

[1] G. C. Goodwin, S. F. Graebe, and M. E. Salgado, "Control system design," Upper Saddle River, 2001.

[2] P. Pivoka, Analysis and Design of Fuzzy PID Controller Based on Classical PID Controller Approach, ser. Fuzzy Control. Springer, 2000, pp. 186-199.

[3] J. I. Corcau, E. Stoenescu, and M. Lungu, "Comparative analysis of classical and fuzzy pi algorithms," in Proceedings of the 7th WSEAS International Conference on Artificial intelligence, knowledge engineering and data bases. World Scientific and Engineering Academy and Society (WSEAS), 2008, pp. 189-192.

[4] K. Craig, "Fuzzy logic and fuzzy control," Report, Rensselaer Polytechnic Institute, 2011.

[5] A. F. Payam, E. M. A. Rahman, and M. Fathipour, Control of Atomic Force Microscope Based on the Fuzzy Theory. INTECH Open Access Publisher, 2011.

[6] A. J. Fleming and K. K. Leang, Design, Modeling and Control of Nanopositioning Systems. Springer, 2014.

[7] S. S. Aphale, A. J. Fleming, and S. O. R. Moheimani, "Inte-gral resonant control of collocated smart structures," Smart Materials and Structures, vol. 16, no. 2, p. 439, 2007.

[8] B. Bhikkaji, M. Ratnam, and S. O. R. Moheimani, "PVPF control of piezoelectric tube scanners," Sensors and Actuators A: Physical, vol. 135, no. 2, pp. 700-712, 2007.

[9] Y. K. Yong, B. Ahmed, and S. O. R. Moheimani, "A 12electrode piezoelectric tube scanner for fast atomic force microscopy," in American Control Conference (ACC), 2010, 2010, pp. 4957-4962.

[10] S. S. Aphale, B. Bhikkaji, and S. O. R. Moheimani, "Minimizing scanning errors in piezoelectric stack-actuated nanoposition-ing platforms," IEEE Transactions on Nanotechnology, vol. 7, no. 1, pp. 79-90, 2008.
[11] Y. R. Teo, D. Russell, S. S. Aphale, and A. J. Fleming, "Optimal integral force feedback and structured pi tracking control: Application for high speed confocal microscopy." IFAC Proceedings Volumes, vol. 47, no. 3, pp. 11 793-11 799, 2014.

[12] M. Namavar, A. J. Fleming, M. Aleyaasin, K. Nakkeeran, and S. S. Aphale, "An analytical approach to integral resonant control of second-order systems," Mechatronics, IEEE/ASME Transactions on, vol. 19, no. 2, pp. 651-659, 2014.

[13] J. Zhou and C. Wen, Adaptive backstepping control of uncertain systems: nonsmooth nonlinearities, interactions or timevariations. Springer, 2008.

[14] Q. Xu and K. K. Tan, Advanced Control of Piezoelectic Micro-/nano-positioning Systems. Springer, 2015.

[15] M. Rakotondrabe, "Bouc-wen modeling and inverse multiplicative structure to compensate hysteresis nonlinearity in piezoelectric actuators." IEEE Transactions on Automation Science and Engineering, vol. 8, no. 2, pp. 428-431, 2011.

[16] G.-R. Yu, C.-S. You, and R.-J. Hong, "Self-tuning fuzzy control of a piezoelectric actuator system," in Systems, Man and Cybernetics, 2006. SMC'06. IEEE International Conference on, vol. 2. IEEE, 2006, pp. 1108-1113.

[17] M. S. Hitam, Design and Implementation of Fuzzy Control for Industrial Robot. INTECH Open Access Publisher, 2006.

[18] H.-X. Li and H. B. Gatland, "Conventional fuzzy control and its enhancement," Systems, Man, and Cybernetics, Part B: Cybernetics, IEEE Transactions on, vol. 26, no. 5, pp. 791797, 1996.

[19] M. Altaher, S. S. Aphale, and D. Russell, "A multiloop control strategy for enhanced nanopositioning," in Modelling and Simulation, vol. 838, 838-001, 2016. [Online]. Available: http://www.actapress.com/Content_Of_ Proceeding.aspx? ProceedingID $=766$

[20] Y. R. Teo, D. Russell, S. S. Aphale, and A. J. Fleming, "Optimal integral force feedback and structured pi tracking control: Application for objective lens positioner," Mechatronics, vol. 24, no. 6, pp. 701-711, 2014.

[21] Y. K. Yong, S. S. Aphale, and S. O. R. Moheimani, "Design, identification, and control of a flexure-based xy stage for fast nanoscale positioning," Nanotechnology, IEEE Transactions on, vol. 8, no. 1, pp. 46-54, 2009.

[22] S. S. Aphale, B. Bhikkaji, and S. O. R. Moheimani, "Minimizing scanning errors in piezoelectric stack-actuated nanopositioning platforms," Nanotechnology, IEEE Transactions on, vol. 7, no. 1, pp. 79-90, 2008.

[23] D. Russell, A. J. Fleming, and S. S. Aphale, "Simultaneous optimization of damping and tracking controller parameters via selective pole placement for enhanced positioning bandwidth of nanopositioners," in American Control Conference (ACC), 2014, 2014, pp. 2184-2189.

[24] I. Mahmood and S. O. R. Moheimani, "Making a commercial atomic force microscope more accurate and faster using positive position feedback control," Review of Scientific Instruments, vol. 80, no. 6, p. 063705, 2009. 